\title{
Noctuid moths (Lepidoptera: Noctuoidea: Nolidae, Erebidae, Noctuidae) of the Nowa Dęba Military Training Area in the Sandomierz Primeval Forest (south-eastern Poland)
}

\author{
Janusz NOWACKI ${ }^{1}$ and Krzysztof PAŁKA ${ }^{2}$ \\ ${ }^{1}$ Department of Entomology and Environmental Protection, Poznań University of Life Sciences, Dąbrowskiego 159, \\ 60-594 Poznań, Poland; e-mail: jnowacki@up.poznan.pl \\ ${ }^{2}$ Institute of Biology, University of Maria Curie-Sklodowska, Akademicka 19, 20-033 Lublin, Poland; \\ e-mail: krzysztof.palka@umcs.lublin.pl
}

\begin{abstract}
The paper presents the results of faunistic research on noctuid moths (Lepidoptera, Noctuoidea: Nolidae, Erebidae, Noctuidae) occurring in the ecosystems of the Nowa Dęba Military Training Area in the Sandomierz Primeval Forest in the years 1999-2013. The study yielded 273 species, i.e. $55 \%$ of the Polish Noctuoidea. Its results clearly indicate that the study area is of crucial importance to the conservation of noctuid biodiversity, not only in Poland, but also in Europe. This area is inhabited by a number of stenotopic species, considered to be rare and threatened with extinction in Poland as well as in central Europe as a whole, e.g.: Idia calvaria, Meganola togatulalis, Diachrysia zosimi, Syngrapha microgamma, Abrostola asclepiadis, Acronicta menyanthidis, Heliothis ononis, Hoplodrina respersa, Lithophane lamda, Xylomoia graminea, Hydraecia ultima, Diarsia florida, Lycophotia molothina, Paradiarsia punicea, Xestia castanea, Coenophila subrosea and Euxoa vitta.
\end{abstract}

Key words: Noctuids, SE Poland, Sandomierz Basin, diversity, hygrophilous and psammophilous species, distribution

\section{INTRODUCTION}

Moths are the group of insects which, in the history of entomological research, has been collected and studied the most often. Moreover, the end of the $20^{\text {th }}$ century witnessed a significant intensification of studies on numerous families of moths, including the Noctuoidea superfamily. Despite this, the areas studied for their noctuids in Poland are very unevenly distributed - a highly unsatisfactory situation. The intensification of research into Noctuidae in Poland has produced many papers, including regional studies focusing solely on the Noctuidae family. At the moment, the eastern part of Poland has been the most intensively studied as regards noctuid moth distribution.

The areas where Noctuidae have been well covered include the Biebrza Marshes (Frąckiel \& Nowacki 2010), the Augustów Primeval Forest (Nowacki \& Rudny 1992, Nowacki 2006), the Białowieża Primeval Forest (Buszko et al. 1996), central Podlasie (Nowacki \& Wasiluk 2004), Polesie (Nowacki \& Hołowiński 1999, Hołowiński \& Nowacki 2014), Roztocze (Nowacki 1992), and the Bieszczady Mountains and Przemyśl Foothills (Bielewicz 1973, 1984). Papers summarizing knowledge of Noctuidae in Poland as a whole have also been published (Nowacki 1994, Buszko \& Nowacki 2000). There are 500 species from the Noctuoidea in Poland - this includes those reported recently.

Although faunistic studies of moths have been conducted in Poland for over 150 years, the Podkarpacie province, with the exception of the Przemyśl Foothills and the Bieszczady Mountains, is among the least known in terms of noctuid occurrence (Buszko \& Nowacki 2000). This also applies to the Sandomierz Basin, with the Sandomierz Primeval Forest, where Nowa Dęba Military Training Area is located. The Noctuidae of the Sandomierz Basin had not been studied before the end of the $20^{\text {th }}$ century. Some information on interesting noctuids in the 
southern parts of the Sandomierz Basin was given by Kulesza (1936), and a full list of 302 noctuid species found in the early $20^{\text {th }}$ century in the Rytwiany area, to the south-west of Sandomierz, was presented by Karpowicz (1928). However, the latter area lies beyond the Sandomierz Basin.

The Sandomierz Basin, particularly its western part, is characterised by a specific set of habitat conditions that govern the occurrence of stenotopic plants and animal species. These include, for example, extensive areas of psammophilous habitats, which in other parts of Poland are usually small and scattered. Insects are of particular interest there as, unlike vertebrates, they include numerous stenotopic species, closely associated with this type of habitat.

From 1999 to 2013 the authors conducted faunistic research in order to determine the noctuid community structure of the Nowa Dęba Military Training Area in the Sandomierz Primeval Forest.

\section{STUDY AREA}

In general terms, the study area covers the Nowa Dęba Military Training Area, which is located in the Sandomierz Primeval Forest, which in turn lies in the western part of the Sandomierz Basin (Fig. 1).

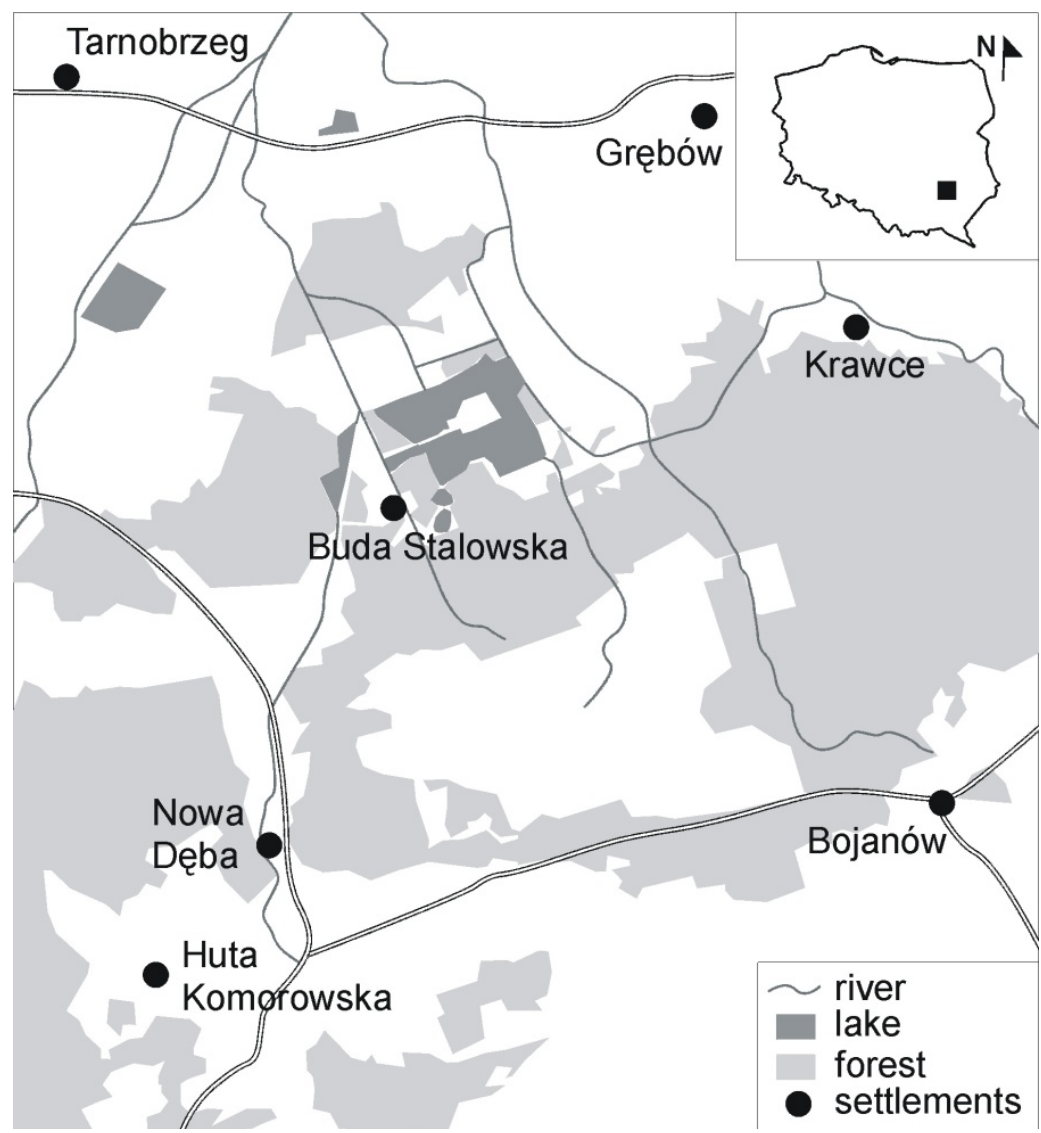

Fig. 1. The location of the Nowa Dęba Military Training Area in Sandomierz Primeval Forest as the research area 
According to the classification adopted by Kondracki (1998), the study area lies in the Western Carpathians - Podkarpacie, the northern Podkarpacie subprovince, the Sandomierz Basin macroregion, and the Tarnobrzeg Plain mesoregion. Triangular in shape, the mesoregion lies between the Vistula valley to the west and the lower San valley to the east, and borders the Kolbuszowa Plateau to the south. The area consists mostly of Pleistocene river sands, which in places form large dune complexes, which can reach heights of $25 \mathrm{~m}$. The Tarnobrzeg Plain is mostly covered with the remains of the Sandomierz Primeval Forest, composed of mixed pine forest with oak trees (Kondracki 1998).

The study area was a military training area situated bordered by the villages of Nowa Dęba, Buda Stalowska, Krawce and Bojanów; it belongs to the Nowa Dęba forestry district. Situated in the Podkarpackie province, it lies in the commune of Bojanów in the district of Stalowa Wola and the communes of Grębów and Nowa Dęba in the district of Tarnobrzeg.

Climatically, the area in question is classified within the Sandomierz region, which covers mainly the Sandomierz Basin. It stands out from the neighbouring regions in that it has more very warm, rain-free days than all of the surrounding areas. The number of warm or cool but overcast days is very much smaller (Woś 1999).

The region's specific geological and climatic conditions resulted in the formation of a plant cover that is often stenotopic. In terms of phytosociology, the communities of psammophilous grasslands on numerous dunes are interesting and varied. The plant communities of the study area can be classified in accordance to their habitat requirements. Starting from the wettest habitats these are as follows:

1. Communities of aquatic plants and bulrushes, mainly in ponds and drainage ditches.

2. Bulrush communities of the Phragmitetea class occurring mainly around the shores and shallow areas of ponds, waterlogged habitats in open areas, scrub and alder forests.

3. Transitional bog communities in the "Cietrzewiec" area.

4. Wetland communities with a high proportion of herbaceous plants, occurring mainly in the western part of the study area.

5. Communities of woodland and willow-alder scrub of the Alnetea glutinosae class, numerous on the periodically flooded wet peat soils, particularly in the north-western part of the study area.

6. Coniferous woodlands of the Vaccinio-Piceetea class, occurring in various forms, depending on the humidity and fertility of the habitat. These are mainly dry, fresh and mixed coniferous woodlands and patches of swampy coniferous forest around the edges of peat bogs.

7. Deciduous woodland communities of the Querco-Fagetea class, which cover a significantly smaller area than the coniferous forests and occur mainly as patches of oak-hornbeam, oak or mixed forests.

8. Psammophilous grasslands and heathland communities, numerous on sunny, sandy dunes.

The study material was regularly collected at the following sites:

- Bojanów, EA68. The study site was located to the west of Bojanów, near the forester's lodge. It was closely surrounded by areas with dominant fresh and wet woodland, as well as patches of segetal and ruderal plant communities.

- Buda Stalowska, EA59. The site was located around the village of Buda Stalowska. The moths were observed in the following habitats: a complex of fish ponds with dominant bulrush communities, on wet meadows at the edge of the village and in the oak-hornbeam woodlands around the village.

- Huta Komorowska, EA48. The site was located next to the forester's lodge in the north of the village of Krawce. The woodlands in its immediate vicinity are deciduous and mixed. 
- Krawce, EA69. The site was located in the north of the study area, near the village of Krawce. The moths were observed in several different habitat types: fresh coniferous woodland, mixed coniferous woodland, deciduous forests with oak, hornbeam and spruce, as well as riparian woodland, marshy forests and synanthropic habitats.

- Nowa Dęba, EA58. The site was located in the central part of the Nowa Dęba Military Training Area within the so-called working area. The moths were observed in several types of habitats. The central area was taken up by a transitional peat bog at the "Cietrzewiec" site, however, the dominant type were the ecosystems that had formed on the extensive dune areas. They included small patches of dry coniferous forest, but were dominated by psammophilous swards and extensive dune heaths, which cover a significant part of the training area.

\section{METHODS}

During the study, observations were carried out in the above areas from 1999 to 2013. Owing to the diversity of habitats in selected sites, the noctuids were caught using various methods, though mainly in light traps. They were caught at night in light traps equipped with $250 \mathrm{~W}$ or $500 \mathrm{~W}$ mercury vapour lamps, powered by a portable generator. In the last year of research the material was collected using many portable light traps equipped with three Philips TL 6W/08 fluorescent lamps, which every night were placed in numerous different ecosystems. The moths were also caught on wine ropes, the bait consisting of fruit juices mixed with wine. The ropes were hung on the branches of trees and shrubs out of range of any lights in the various plant communities. Moths were also caught in the evenings over blooming plants. A small number of moths were collected on sight during the day.

\section{RESULTS}

The study conducted at the Nowa Dęba Military Training Area yielded 273 species of moths from the families Nolidae, Erebidae and Noctuidae. The table below shows all the species of Noctuoidea reported from the study area in a systematic order adopted from Fibiger \& Hacker (2005) (Tab. 1).

Tab. 1. Systematic list of noctuid moths (Nolidae, Erebidae, Noctuidae) recorded in the Nowa Dęba Military Training Area in the Sandomierz Primeval Forest from 1999 to 2013; study sites: 1 - Buda Stalowska, 2 - Krawce, 3 Bojanów, 4 - Huta Komorowska, 5 - Nowa Dęba.

* Roman numerals denote months, Arabic numerals indicates the decade of the month i.e.: (1) - from the 1 st to the 10 th day of each month, (2) - from the 11th to the 20th day; (3) - from 21st to 30/31 day.

** Species discussed separately.

\begin{tabular}{|c|c|c|c|c|c|c|c|}
\hline \multirow[b]{2}{*}{ No. } & \multirow[b]{2}{*}{ Species } & \multicolumn{5}{|c|}{ Study site } & \multirow[b]{2}{*}{ Flight period* } \\
\hline & & 1 & 2 & 3 & 4 & 5 & \\
\hline \multicolumn{8}{|c|}{ Herminiinae } \\
\hline 1 & Idia calvaria (Denis \& Schiff., 1775) ** & $\mathrm{x}$ & & & & & 9 VI 2013 \\
\hline 2 & Paracolax tristalis (Fabricius, 1794) & $\mathrm{x}$ & $\mathrm{x}$ & $\mathrm{x}$ & $\mathrm{x}$ & $\mathrm{x}$ & VI (2)-VIII(1) \\
\hline 3 & Herminia tarsicrinalis (Knoch, 1782) & $\mathrm{x}$ & $\mathrm{x}$ & & & $\mathrm{x}$ & VI(2), VII(1)-VIII(2) \\
\hline 4 & Herminia grisealis (Denis et Schiff., 1775) & $\mathrm{x}$ & $\mathrm{x}$ & & $\mathrm{x}$ & & VI(1-2), VII(2) \\
\hline 5 & Pechipogo strigillata (Linnaeus, 1758) & $\mathrm{x}$ & & & & $\mathrm{x}$ & $\mathrm{VI}(1-2)$ \\
\hline \multicolumn{8}{|c|}{ Hypeninae } \\
\hline 6 & Hypena proboscidalis (Linnaeus, 1758) & $\mathrm{x}$ & $\mathrm{x}$ & $\mathrm{x}$ & $\mathrm{x}$ & $\mathrm{x}$ & VI(1-2), VII(3)-X(3) \\
\hline 7 & Hypena rostralis (Linnaeus, 1758) & $\mathrm{x}$ & $\mathrm{x}$ & $\mathrm{x}$ & $\mathrm{x}$ & $\mathrm{x}$ & $\mathrm{V}(1)-\mathrm{VI}(2), \mathrm{VIII}(1)-\mathrm{X}(3)$ \\
\hline 8 & Hypena crassalis (Fabricius, 1787) & $\mathrm{x}$ & $\mathrm{x}$ & $\mathrm{x}$ & $\mathrm{x}$ & $\mathrm{x}$ & VI(1)-VII(3) \\
\hline \multicolumn{8}{|c|}{ Rivulinae } \\
\hline 9 & Rivula sericealis (Scopoli, 1763) & $\mathrm{x}$ & $\mathrm{x}$ & $\mathrm{x}$ & $\mathrm{x}$ & $\mathrm{x}$ & VI(1-2), VII(2)-X(3) \\
\hline
\end{tabular}


Study site

\begin{tabular}{lllll}
\multicolumn{5}{c}{ Study site } \\
\hline 1 & 2 & 3 & 4 & 5
\end{tabular}

\section{Scoliopteryginae}

10 Scoliopteryx libatrix (Linnaeus, 1758)

\section{Hypenodinae}

11 Hypenodes humidalis (Doubleday, 1850)

12 Schrankia costaestrigalis (Stephens, 1834)

13 Schrankia taenialis (Hübner, 1809)

\section{Aventiinae}

14 Laspeyria flexula (Denis \& Schiff., 1775)

\section{Phytometrinae}

15 Colobochyla salicalis (Denis \& Schiff., 1775)

16 Trisateles emortualis (Denis et Schiff., 1775)

\section{Catocalinae}

17 Catocala fraxini (Linnaeus, 1758)

18 Catocala sponsa (Linnaeus, 1767)

19 Catocala promissa (Denis \& Schiff., 1775)

20 Catocala nupta (Linnaeus, 1767)

21 Catocala electa (Vieweg, 1790)

22 Catocala fulminea (Scopoli, 1763)

23 Minucia lunaris (Denis \& Schiff., 1775)

24 Lygephila pastinum (Treitschke, 1826)

25 Lygephila viciae (Hübner, 1822)

26 Euclidia mi (Clerck, 1759)

27 Euclidia glyphica (Linnaeus, 1758)

\section{Nolinae}

28 Meganola togatulalis (Hübner, 1796) **

29 Meganola strigula (Denis \& Schiff., 1775)

30 Meganola albula (Denis \& Schiff., 1775)

31 Nola confusalis (Herrich-Schäffer, 1847)

32 Nola cicatricalis (Treitschke, 1835)

33 Nola aerugula (Hübner, 1793)

\section{Eariadinae}

34 Earias clorana (Linnaeus, 1761)

\section{Chloephorinae}

35 Nycteola revayana (Scopoli, 1772)

36 Nycteola degenerana (Hübner, 1799)

37 Bena bicolorana (Fuessly, 1775)

38 Pseudoips prasinana (Linnaeus, 1758)

\section{Plusiinae}

39 Diachrysia chrysitis (Linnaeus, 1758)

40 Diachrysia stenochrysis (Warren, 1913)

41 Diachrysia zosimi (Hübner, 1822) **

42 Macdunnoughia confusa (Stephens, 1850)

43 Plusia festucae (Linnaeus, 1758)

44 Plusia putnami (Grote, 1873)

45 Autographa gamma (Linnaeus, 1758)

46 Autographa pulchrina (Haworth, 1809)

47 Autographa buraetica (Staudinger, 1892)

49 Syngrapha microgamma (Hübner, 1823) **

\begin{tabular}{|c|c|c|c|}
\hline$X$ & $\mathrm{X}$ & & $\mathrm{X}$ \\
\hline \multirow[t]{2}{*}{$X$} & $\mathrm{X}$ & & \\
\hline & $\mathrm{X}$ & $\mathrm{X}$ & $\mathrm{X}$ \\
\hline \multicolumn{4}{|l|}{$X$} \\
\hline $\mathrm{X}$ & $\mathrm{X}$ & $\mathrm{X}$ & $\mathrm{X}$ \\
\hline$X$ & $\mathrm{X}$ & & \\
\hline$X$ & $\mathrm{X}$ & $X$ & \\
\hline $\mathrm{X}$ & $\mathrm{X}$ & & $\mathrm{X}$ \\
\hline \multicolumn{4}{|c|}{$X$} \\
\hline$X$ & $\mathrm{X}$ & & $\mathrm{X}$ \\
\hline & & & $\mathrm{X}$ \\
\hline \multirow[t]{2}{*}{$X$} & $\mathrm{X}$ & $X$ & \\
\hline & $\mathrm{X}$ & & \\
\hline $\mathrm{X}$ & $\mathrm{X}$ & & \\
\hline $\mathrm{X}$ & $\mathrm{X}$ & & \\
\hline $\mathrm{X}$ & & & \\
\hline
\end{tabular}

V(1), VII(1)-X(3)

VI(1), VIII(1-2)

VII(3)-X(3)

IX(1)

VI(1-2), VII(1)-X(3)

VI(1-2)

X VII(1)-VIII(2)

$\mathrm{x}$

VIII(1)-IX(3)

VII(2)-IX(3)

VII(2)-VIII(2)

VIII(3)-IX(1)

VII(3)-IX(2)

VII(1)-VIII(1)

$\mathrm{V}(1-2)$

VI(2), VIII(1)-IX(1)

$\mathrm{VI}(1-2)$

$\mathrm{V}(2)$

$\mathrm{V}(2-3)$

$\mathrm{X}$

VII(1-3)

$\mathrm{VI}(2)$

VII(1-3)

IV(3)-V(1)

$\operatorname{IV}(3)$

VI(3)- VII(3), IX(3)

VII(1)-VIII(1)

VIII(3)-IX(1)

$\mathrm{X}(3)$

VI(1)-VIII(1)

VI(2)-VII(1)

VI(1)-VII(3), VIII(1)-IX(2)

VI(1)-VII(3),VIII(1)-IX(2)

18. VI. 2013

VI(1) - IX(2)

$\mathrm{VI}(1)-\mathrm{VIII}(2)$

VIII(1-2)

V(1), VII(1)-IX(2)

VI(1)-VII(3)

5 VII 2013, 1 ex.

VI(2) 


\begin{tabular}{|c|c|c|c|c|c|c|c|}
\hline \multirow[b]{2}{*}{ No. } & \multirow[b]{2}{*}{ Species } & \multicolumn{5}{|c|}{ Study site } & \multirow[b]{2}{*}{ Flight period* } \\
\hline & & 1 & 2 & 3 & 4 & 5 & \\
\hline 50 & Abrostola tripartita (Hufnagel, 1766) & $\mathrm{x}$ & $\mathrm{x}$ & & $\mathrm{x}$ & $\mathrm{x}$ & VI(1-2), VIII(1)-IX(1) \\
\hline 51 & Abrostola asclepiadis (Denis \& Schiff., 1775) ** & & & & & $\mathrm{x}$ & $\mathrm{VI}(1)$ \\
\hline \multirow[t]{2}{*}{52} & Abrostola triplasia (Linnaeus, 1758) & $\mathrm{x}$ & $\mathrm{x}$ & $\mathrm{x}$ & $\mathrm{x}$ & $\mathrm{x}$ & VI(1-3), VII(2)-IX(1) \\
\hline & Eustrotiinae & & & & & & \\
\hline 53 & Deltote pygarga (Hufnagel, 1766) & $\mathrm{x}$ & $\mathrm{x}$ & $\mathrm{x}$ & $\mathrm{x}$ & $\mathrm{x}$ & VI(1)-VII(3), IX(1) \\
\hline 54 & Deltote deceptoria (Scopoli, 1763) & & & & & $\mathrm{x}$ & $\mathrm{VI}(1)$ \\
\hline 55 & Deltote uncula (Clerck, 1759) & $\mathrm{x}$ & & & & $\mathrm{x}$ & VI(1), VII(3)-VIII(1) \\
\hline \multirow[t]{2}{*}{56} & Deltote bankiana (Fabricius, 1775) & $\mathrm{x}$ & & & $\mathrm{x}$ & $\mathrm{x}$ & VI(1), VII(1-3) \\
\hline & Pantheinae & & & & & & \\
\hline 57 & Panthea coenobita (Esper, 1785) & $\mathrm{x}$ & $\mathrm{x}$ & $\mathrm{x}$ & $\mathrm{x}$ & $\mathrm{x}$ & VI(1)-VIII(1) \\
\hline 58 & $\begin{array}{l}\text { Colocasia coryli (Linnaeus, 1758) } \\
\text { Dilobinae }\end{array}$ & $\mathrm{x}$ & $\mathrm{x}$ & $\mathrm{x}$ & $\mathrm{x}$ & $\mathrm{x}$ & VI(1-3), VII(2)-VIII(2) \\
\hline \multirow[t]{2}{*}{59} & Diloba caeruleocephala (Linnaeus, 1758) & $\mathrm{x}$ & $\mathrm{x}$ & & & $\mathrm{x}$ & $\operatorname{IX}(2)-X(3)$ \\
\hline & Acronictinae & & & & & & \\
\hline 60 & Moma alpium (Osbeck, 1778) & $\mathrm{x}$ & $\mathrm{x}$ & & $\mathrm{x}$ & $\mathrm{x}$ & VI(1)-VII(2), VIII(1) \\
\hline 61 & Acronicta alni (Linnaeus, 1767) & $\mathrm{x}$ & $\mathrm{x}$ & $\mathrm{x}$ & $\mathrm{x}$ & $\mathrm{x}$ & V(1)-VII(1), VIII(1) \\
\hline 62 & Acronicta cuspis (Hübner, 1813) & & $\mathrm{x}$ & $\mathrm{x}$ & $\mathrm{x}$ & $\mathrm{x}$ & VI(2)-VII(1), VIII(1) \\
\hline 63 & Acronicta psi (Linnaeus, 1758) & $\mathrm{x}$ & $\mathrm{x}$ & & & $\mathrm{x}$ & VI(1-2), VIII(1) \\
\hline 64 & Acronicta aceris (Linnaeus, 1758) & $\mathrm{x}$ & & & & $\mathrm{x}$ & $\mathrm{V}(2)-\mathrm{VI}(3), \mathrm{VII}(1-3)$ \\
\hline 65 & Acronicta leporina (Linnaeus, 1758) & $\mathrm{x}$ & $\mathrm{x}$ & & & $\mathrm{x}$ & VI(1-2), VII(1)-VIII(2) \\
\hline 66 & Acronicta megacephala (Denis et Schiff., 1775) & $\mathrm{x}$ & & $\mathrm{x}$ & $\mathrm{x}$ & $\mathrm{x}$ & $\mathrm{V}(1)-\mathrm{VI}(3), \mathrm{VII}(2)-\mathrm{VIII}(2)$ \\
\hline 67 & Acronicta strigosa (Denis et Schiff. 1775) & $\mathrm{x}$ & $\mathrm{x}$ & & $\mathrm{x}$ & $\mathrm{x}$ & VI(2)-VII(3) \\
\hline 68 & Acronicta menyanthidis (Esper, 1789) ** & & & $\mathrm{x}$ & & $\mathrm{x}$ & VII(1-2) \\
\hline 69 & Acronicta auricoma (Denis et Schiff., 1775) & $\mathrm{x}$ & $\mathrm{x}$ & $\mathrm{x}$ & & $\mathrm{x}$ & VI(1), VII(1)-VIII(1) \\
\hline 70 & Acronicta cinerea (Hufnagel, 1766) & & & $\mathrm{x}$ & & $\mathrm{x}$ & V(1), VII(3)-VIII(1) \\
\hline 71 & Acronicta rumicis (Linnaeus, 1758) & $\mathrm{x}$ & $\mathrm{x}$ & $\mathrm{x}$ & $\mathrm{x}$ & $\mathrm{x}$ & VII(1)-VIII(2) \\
\hline 72 & Craniophora ligustri (Denis et Schiff., 1775) & $\mathrm{x}$ & $\mathrm{x}$ & & $\mathrm{x}$ & $\mathrm{x}$ & VI(1-2), VII(2) -VIII(2) \\
\hline 73 & Simyra nervosa (Denis et Schiff., 1775) & & & & & $\mathrm{x}$ & 24 VII 2013 \\
\hline \multirow[t]{2}{*}{74} & Simyra albovenosa (Goeze, 1781) & $\mathrm{x}$ & $\mathrm{x}$ & & & $\mathrm{x}$ & V(1), VII(1)-VIII(1) \\
\hline & Cuculliinae & & & & & & \\
\hline 75 & Cucullia artemisiae (Hufnagel, 1766) & $\mathrm{x}$ & & & & & VII(1) \\
\hline 76 & Cucullia umbratica (Linnaeus, 1758) & & & & $\mathrm{x}$ & $\mathrm{x}$ & $\mathrm{VI}(1)-\mathrm{VII}(1)$ \\
\hline \multirow[t]{2}{*}{77} & Shargacucullia verbasci (Linnaeus, 1758) & $\mathrm{x}$ & & & & & VI(1) \\
\hline & Amphipyrinae & & & & & & \\
\hline 78 & Amphipyra pyramidea (Linnaeus, 1758) & $\mathrm{x}$ & $\mathrm{x}$ & & $\mathrm{x}$ & $\mathrm{x}$ & $\mathrm{VII}(2)-\mathrm{X}(3)$ \\
\hline 79 & Amphipyra berbera Rungs, 1949 & $\mathrm{x}$ & $\mathrm{x}$ & & $\mathrm{x}$ & $\mathrm{x}$ & VII(1)-X(3) \\
\hline 80 & Amphipyra livida (Denis \& Schiff., 1775) & $\mathrm{x}$ & $\mathrm{x}$ & & $\mathrm{x}$ & $\mathrm{x}$ & VIII(1)-X(3) \\
\hline \multirow[t]{2}{*}{81} & Amphipyra tragopoginis (Clerck, 1759) & & & & & $\mathrm{x}$ & VIII(3)-IX(1) \\
\hline & Psaphidinae & & & & & & \\
\hline 82 & Asteroscopus sphinx (Hufnagel, 1766) & & $\mathrm{x}$ & & $\mathrm{x}$ & & $\mathrm{X}(3)$ \\
\hline 83 & Brachionycha nubeculosa (Esper, 1785) & $\mathrm{x}$ & $\mathrm{x}$ & $\mathrm{x}$ & $\mathrm{x}$ & $\mathrm{x}$ & $\operatorname{IV}(2)$ \\
\hline \multirow[t]{2}{*}{84} & Allophyes oxyacanthae (Linnaeus, 1758) & $\mathrm{x}$ & $\mathrm{x}$ & $\mathrm{x}$ & $\mathrm{x}$ & $\mathrm{x}$ & $\operatorname{IX}(2)-X(3)$ \\
\hline & Oncocnemidinae & & & & & & \\
\hline \multirow[t]{2}{*}{85} & Calophasia lunula (Hufnagel, 1766) & & & & & $\mathrm{x}$ & $\mathrm{VI}(1)$ \\
\hline & Condicinae & & & & & & \\
\hline 86 & Eucarta virgo (Treitschke, 1835) & $\mathrm{x}$ & & & $\mathrm{x}$ & $\mathrm{x}$ & VI(1), VII(1)-VIII(1) \\
\hline
\end{tabular}


Study site

\begin{tabular}{llcrr}
\multicolumn{5}{c}{ Study site } \\
\hline 1 & 2 & 3 & 4 & 5
\end{tabular}

Flight period*

\section{Heliothinae}

87 Protoschinia scutosa (Denis \& Schiff., 1775)

88 Heliothis viriplaca (Hufnagel, 1766)

89 Heliothis adaucta (Butler, 1878)

$=$ Heliothis maritima auct.

90 Heliothis ononis (Denis \& Schiff., 1775) **

91 Helicoverpa armigera (Hübner, 1808)

92 Pyrrhia umbra (Hufnagel, 1766)

\section{Eriopinae}

93 Callopistria juventina (Stoll, 1782)

\section{Bryophilinae}

94 Cryphia fraudatricula (Hübner, 1803)

95 Cryphia algae (Fabricius, 1775)

\section{Xyleninae}

96 Caradrina morpheus (Hufnagel, 1766)

97 Paradrina selini (Boisduval, 1840)

98 Paradrina clavipalpis (Scopoli, 1763)

99 Hoplodrina octogenaria (Goeze, 1781)

100 Hoplodrina blanda (Denis \& Schiff., 1775)

101 Hoplodrina respersa (Denis \& Schiff., 1775) **

102 Hoplodrina ambigua (Denis \& Schiff., 1775)

103 Chilodes maritima (Tauscher, 1806)

104 Athetis lepigone (Möschler, 1860)

105 Athetis pallustris (Hübner, 1808)

106 Charanyca trigrammica (Hufnagel, 1766)

107 Charanyca ferruginea (Esper, 1785)

108 Dypterygia scabriuscula (Linnaeus, 1758)

109 Thalpophila matura (Hufnagel, 1766)

110 Trachea atriplicis (Linnaeus, 1758)

111 Euplexia lucipara (Linnaeus, 1758)

112 Phlogophora meticulosa (Linnaeus, 1758)

113 Hyppa rectilinea (Esper, 1788)

114 Actinotia polyodon (Clerck, 1759)

115 Elaphria venustula (Hübner, 1790)

116 Pseudeustrotia candidula (Denis \& Schiff.,1775)

117 Ipimorpha retusa (Linnaeus, 1761)

118 Ipimorpha subtusa (Denis \& Schiff., 1775)

119 Enargia paleacea (Esper, 1788)

120 Cosmia pyralina (Denis \& Schiff., 1775)

121 Cosmia trapezina (Linnaeus, 1758)

122 Xanthia togata (Esper, 1788)

123 Xanthia icteritia (Hufnagel, 1766)

124 Xanthia gilvago (Denis \& Schiff., 1775)

125 Tiliacea citrago (Linnaeus, 1758)

126 Agrochola circellaris (Hufnagel, 1766)

127 Agrochola lota (Clerck, 1759)

128 Agrochola macilenta (Hübner, 1809)

129 Agrochola nitida (Denis \& Schiff., 1775)

130 Agrochola helvola (Linnaeus, 1758)
$\mathrm{X}$

X

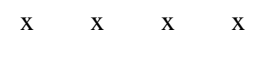

$\mathrm{X}$

$\mathrm{X}$

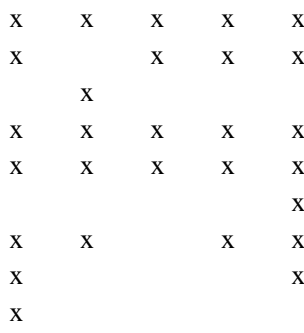

$\mathrm{x}$

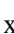

$\mathrm{x}$

$\mathrm{X}$

$\mathrm{x}$

$\mathrm{X}$

$\mathrm{x}$

$\mathrm{X}$

$\mathrm{x}$

$\mathrm{x} \quad \mathrm{X}$
VII(2-3)

VI(1), VII(3)

$$
\begin{gathered}
\text { VII(3)-VIII(1) } \\
\text { VI(1-2) }
\end{gathered}
$$

VIII(1)-IX(1)

VI(2), VII(2)-VIII(2)

VI(1), VII(3)-VIII91)

VI(1)

\begin{tabular}{|c|}
\hline VI(1)-VIII(2) \\
\hline VI(1)-VII(2) \\
\hline VIII(2) \\
\hline VI(2)-VII(3) \\
\hline VII(1-3) \\
\hline 3 VIII 2013 \\
\hline VI(2)-IX(1) \\
\hline VI(2)-VIII(2) \\
\hline VIII(1) \\
\hline VII(1-2) \\
\hline $\mathrm{V}(2)-\mathrm{VI}(3)$ \\
\hline VI(1)-VII(3) \\
\hline VI(1) - VIII(3) \\
\hline VIII(1-3) \\
\hline VI(1)-VII(3), VIII(3)-IX(1) \\
\hline VI(1)-VII(1) \\
\hline V(1-2), VIII(2)-X(3) \\
\hline V(2)-VIII(1) \\
\hline V(2)-VIII(2) \\
\hline VI(1)-VIII(3) \\
\hline $\mathrm{V}(2)-\mathrm{VI}(2), \mathrm{VIII}(1-2)$ \\
\hline VII(1-3) \\
\hline VII(1)-VIII(3) \\
\hline VII(2)-IX(2) \\
\hline VI(3)-VII(3) \\
\hline VI(3)-IX(1) \\
\hline $\operatorname{IX}(2)-X(2)$ \\
\hline VIII(2)-X(3) \\
\hline 6 Oct 2013 \\
\hline IX(3)-X(3) \\
\hline VIII(2)-X(3) \\
\hline $\mathrm{X}(3)$ \\
\hline$X(2-3)$ \\
\hline $\mathrm{IX}(1)-\mathrm{X}(2)$ \\
\hline IX(2)-X(3) \\
\hline
\end{tabular}

VIII(2) 


\begin{tabular}{|c|c|c|c|c|c|c|c|}
\hline \multirow[b]{2}{*}{ No. } & \multirow[b]{2}{*}{ Species } & \multicolumn{5}{|c|}{ Study site } & \multirow[b]{2}{*}{ Flight period* } \\
\hline & & 1 & 2 & 3 & 4 & 5 & \\
\hline 131 & Agrochola litura (Linnaeus, 1758) & $\mathrm{x}$ & $\mathrm{x}$ & & $\mathrm{x}$ & $\mathrm{x}$ & $X(2-3)$ \\
\hline 132 & Eupsilia transversa (Hufnagel, 1766) & $\mathrm{x}$ & $\mathrm{x}$ & $\mathrm{x}$ & $\mathrm{x}$ & $\mathrm{x}$ & IV(2), IX(3)-X(3) \\
\hline 133 & Conistra vaccinii (Linnaeus, 1761) & $\mathrm{x}$ & $\mathrm{x}$ & $\mathrm{x}$ & $\mathrm{x}$ & $\mathrm{x}$ & $\operatorname{IV}(2)-V(1), \operatorname{IX}(2)-X(3)$ \\
\hline 134 & Conistra ligula (Esper, 1791) & & $\mathrm{x}$ & & $\mathrm{x}$ & $\mathrm{x}$ & $\operatorname{IV}(2), X(2)$ \\
\hline 135 & Conistra rubiginosa (Scopoli, 1763) & & $\mathrm{x}$ & & & & 26 IV 2013 \\
\hline 136 & Conistra rubiginea (Denis \& Schiff., 1775) & $\mathrm{x}$ & $\mathrm{x}$ & $\mathrm{x}$ & $\mathrm{x}$ & $\mathrm{x}$ & $\operatorname{IV}(2)-V(1), X(2)$ \\
\hline 137 & Conistra erythrocephala (Denis \& Schiff., 1775) & $\mathrm{x}$ & & & $\mathrm{x}$ & & $\operatorname{IV}(2)$ \\
\hline 138 & Lithomoia solidaginis (Hübner, 1803) & & $\mathrm{x}$ & $\mathrm{x}$ & $\mathrm{x}$ & $\mathrm{x}$ & VIII(2)-IX(3) \\
\hline 139 & Lithophane socia (Hufnagel, 1766) & & & & & $\mathrm{x}$ & $\mathrm{IX}(1)$ \\
\hline 140 & Lithophane ornitopus (Hufnagel, 1766) & $\mathrm{x}$ & $\mathrm{x}$ & & $\mathrm{x}$ & $\mathrm{x}$ & $\mathrm{IV}(2)-\mathrm{V}(1)$ \\
\hline 141 & Lithophane furcifera (Hufnagel, 1766) & $\mathrm{x}$ & $\mathrm{x}$ & & & $\mathrm{x}$ & $\operatorname{IV}(2)$ \\
\hline 142 & Lithophane lamda (Fabricius, 1787) ** & & $\mathrm{x}$ & & & $\mathrm{x}$ & $\operatorname{IV}(2-3)$ \\
\hline 143 & Parastichtis suspecta (Hübner, 1817) & $\mathrm{x}$ & $\mathrm{x}$ & & & $\mathrm{x}$ & VI(3)-VII(3) \\
\hline 144 & Apterogenum ypsillon (Denis \& Schiff., 1775) & & & & & $\mathrm{x}$ & VI(3) \\
\hline 145 & Griposia aprilina (Linnaeus, 1758) & $\mathrm{x}$ & $\mathrm{x}$ & & & $\mathrm{x}$ & $X(1-2)$ \\
\hline 146 & Dichonia convergens (Denis \& Schiff., 1775) & & & & & $\mathrm{x}$ & $26 \times 2013$ \\
\hline 147 & Mesogona oxalina (Hübner, 1803) & & & & $\mathrm{x}$ & $\mathrm{x}$ & VIII(3)-IX(2) \\
\hline 148 & Ammoconia caecimacula (Denis \& Schiff.,1775) & & $\mathrm{x}$ & & & $\mathrm{x}$ & $X(1-3)$ \\
\hline 149 & Mniotype satura (Denis \& Schiff., 1775) & $\mathrm{x}$ & $\mathrm{x}$ & $\mathrm{x}$ & $\mathrm{x}$ & $\mathrm{x}$ & VIII(3)-X(3) \\
\hline 150 & Apamea monoglypha (Hufnagel, 1766) & $\mathrm{x}$ & $\mathrm{x}$ & $\mathrm{x}$ & $\mathrm{x}$ & $\mathrm{x}$ & VI(1)-VIII(3) \\
\hline 151 & Apamea crenata (Hufnagel, 1766) & & & & & $\mathrm{x}$ & $\mathrm{VI}(1-3)$ \\
\hline 152 & Apamea epomidion (Haworth, 1809) & $\mathrm{x}$ & & & & & VII(1-2) \\
\hline 153 & Apamea lateritia (Hufnagel, 1766) & & & $\mathrm{x}$ & $\mathrm{x}$ & $\mathrm{x}$ & VI(2)-VII(3) \\
\hline 154 & Apamea remissa (Hübner, 1809) & $\mathrm{x}$ & $\mathrm{x}$ & & $\mathrm{x}$ & $\mathrm{x}$ & VI(1)-VII(2) \\
\hline 155 & Apamea unanimis (Hübner, 1813) & $\mathrm{x}$ & & & & $\mathrm{x}$ & $\mathrm{VI}(1-3)$ \\
\hline 156 & Apamea anceps (Denis \& Schiff., 1775) & $\mathrm{x}$ & $\mathrm{x}$ & $\mathrm{x}$ & $\mathrm{x}$ & $\mathrm{x}$ & VI(2)-VII(2) \\
\hline 157 & Apamea sordens (Hufnagel, 1766) & $\mathrm{x}$ & & $\mathrm{x}$ & $\mathrm{x}$ & $\mathrm{x}$ & $\mathrm{VI}(1-3)$ \\
\hline 158 & Apamea scolopacina (Esper, 1788) & $\mathrm{x}$ & $\mathrm{x}$ & & & $\mathrm{x}$ & VI(3)-VII(2) \\
\hline 159 & Lateroligia ophiogramma (Esper, 1794) & $\mathrm{x}$ & & & & $\mathrm{x}$ & VI(2)-VIII(1) \\
\hline 160 & Oligia strigilis (Linnaeus, 1758) & $\mathrm{x}$ & & $\mathrm{x}$ & $\mathrm{x}$ & $\mathrm{x}$ & VI(1)-VII(3) \\
\hline 161 & Oligia latruncula (Denis \& Schiff., 1775) & $\mathrm{x}$ & & $\mathrm{x}$ & $\mathrm{x}$ & $\mathrm{x}$ & $\mathrm{VI}(1-3)$ \\
\hline 162 & Oligia versicolor (Borkhausen, 1792) & $\mathrm{x}$ & $\mathrm{x}$ & & & $\mathrm{x}$ & $\mathrm{VI}(1)-\mathrm{VII}(2)$ \\
\hline 163 & Mesoligia furuncula (Denis \& Schiff., 1775) & $\mathrm{x}$ & $\mathrm{x}$ & $\mathrm{x}$ & & $\mathrm{x}$ & VII(1)-IX(1) \\
\hline 164 & Mesapamea secalis (Linnaeus, 1758) & $\mathrm{x}$ & $\mathrm{x}$ & $\mathrm{x}$ & & $\mathrm{x}$ & VII(1)-VIII(3) \\
\hline 165 & Mesapamea secalella Remm, 1983 & $\mathrm{x}$ & $\mathrm{x}$ & $\mathrm{x}$ & & $\mathrm{x}$ & $\operatorname{VIII}(2-3)$ \\
\hline 166 & Xylomoia graminea (Graeser, 1889) ** & & & & & $\mathrm{x}$ & $\mathrm{VI}(1)$ \\
\hline 167 & Luperina testacea (Denis \& Schiff., 1775) & $\mathrm{x}$ & $\mathrm{x}$ & & $\mathrm{x}$ & $\mathrm{x}$ & VIII $(2-3)$ \\
\hline 168 & Amphipoea oculea (Linnaeus, 1761) & $\mathrm{x}$ & $\mathrm{x}$ & $\mathrm{x}$ & $\mathrm{x}$ & $\mathrm{x}$ & VII(1)-VIII(2) \\
\hline 169 & Amphipoea fucosa (Freyer, 1830) & $\mathrm{x}$ & $\mathrm{x}$ & & $\mathrm{x}$ & $\mathrm{x}$ & VII(2)-VIII(2) \\
\hline 170 & Amphipoea lucens (Freyer, 1845) & $\mathrm{x}$ & $\mathrm{x}$ & & & $\mathrm{x}$ & VII(3)-VIII(2) \\
\hline 171 & Hydraecia micacea (Esper, 1789) & $\mathrm{x}$ & $\mathrm{x}$ & & $\mathrm{x}$ & $\mathrm{x}$ & VII(3)-X(3) \\
\hline 172 & Hydraecia ultima (Holst, 1965) ** & $\mathrm{x}$ & $\mathrm{x}$ & $\mathrm{x}$ & & & VII(1)-VIII(1) \\
\hline 173 & Gortyna flavago (Denis \& Schiff., 1775) & $\mathrm{x}$ & $\mathrm{x}$ & & $\mathrm{x}$ & & VIII(2)-IX(2) \\
\hline 174 & Calamia tridens (Hufnagel, 1766) & & $\mathrm{x}$ & $\mathrm{X}$ & $\mathrm{x}$ & $\mathrm{x}$ & VII(1)-VIII(2) \\
\hline 175 & Helotropha leucostigma (Hübner, 1808) & $\mathrm{x}$ & & & $\mathrm{x}$ & $\mathrm{x}$ & VII(2)-VIII(1) \\
\hline 176 & Rhizedra lutosa (Hübner, 1803) & $\mathrm{x}$ & $\mathrm{x}$ & & & $\mathrm{x}$ & $X(2-3)$ \\
\hline 177 & Nonagria typhae (Thunberg, 1784) & & & & & $\mathrm{x}$ & VII(3)-X(3) \\
\hline 178 & Phragmitiphila nеха (Hübner, 1808) & & $\mathrm{x}$ & $\mathrm{x}$ & & & VIII(3)-IX(2) \\
\hline 179 & Archanara dissoluta (Treitschke, 1825) & $\mathrm{x}$ & & & & $\mathrm{x}$ & VII(1-3) \\
\hline 180 & Capsula sparganii (Esper, 1790) & $\mathrm{x}$ & & & $\mathrm{x}$ & & VII(2)-VIII(3) \\
\hline
\end{tabular}


Study site

\begin{tabular}{|c|c|c|c|c|c|c|c|}
\hline \multirow[b]{2}{*}{ No. } & \multirow[b]{2}{*}{ Species } & & \multirow[b]{2}{*}{ Flight period* } \\
\hline & & 1 & 2 & 3 & 4 & 5 & \\
\hline 181 & Capsula algae (Esper, 1789) & & & & & $\mathrm{x}$ & VIII(3) \\
\hline 182 & Lenisa geminipuncta (Haworth, 1809) & $\mathrm{x}$ & & & & $\mathrm{x}$ & VII(3)-VIII(1) \\
\hline 183 & Sedina buettneri (Hering, 1858) & & $\mathrm{x}$ & & & $\mathrm{x}$ & $\mathrm{IX}(2)-X(3)$ \\
\hline 184 & Photedes fluxa (Hübner, 1809) & $\mathrm{x}$ & $\mathrm{x}$ & $\mathrm{x}$ & $\mathrm{x}$ & $\mathrm{x}$ & VII(1-3) \\
\hline \multirow[t]{2}{*}{185} & Denticucullus pygmina (Haworth, 1809) & $\mathrm{x}$ & $\mathrm{x}$ & $\mathrm{x}$ & $\mathrm{x}$ & $\mathrm{x}$ & VIII(2)-X(3) \\
\hline & Hadeninae & & & & & & \\
\hline 186 & Anarta myrtilli (Linnaeus, 1761) & & $\mathrm{x}$ & & & $\mathrm{x}$ & V(1), VII(3)-VIII(2) \\
\hline 187 & Anarta trifolii (Hufnagel, 1766) & $\mathrm{x}$ & $\mathrm{x}$ & $\mathrm{x}$ & & $\mathrm{x}$ & $\mathrm{V}(1)-\mathrm{IX}(1)$ \\
\hline 188 & Lacanobia w-latinum (Hufnagel, 1766) & $\mathrm{x}$ & $\mathrm{x}$ & & $\mathrm{x}$ & $\mathrm{x}$ & $\mathrm{VI}(1-3)$ \\
\hline 189 & Lacanobia aliena (Hübner, 1808) & $\mathrm{x}$ & & & & $\mathrm{x}$ & VI(2-3), VIII(2) \\
\hline 190 & Lacanobia splendens (Hübner, 1808) & $\mathrm{x}$ & $\mathrm{x}$ & & & $\mathrm{x}$ & $\mathrm{VI}(2)-\mathrm{IX}(1)$ \\
\hline 191 & Lacanobia oleracea (Linnaeus, 1758) & $\mathrm{x}$ & $\mathrm{x}$ & & $\mathrm{x}$ & $\mathrm{x}$ & VI(1)-VIII(3) \\
\hline 192 & Lacanobia thalassina (Hufnagel, 1766) & $\mathrm{x}$ & $\mathrm{x}$ & $\mathrm{x}$ & $\mathrm{x}$ & $\mathrm{x}$ & V(1)-VI(3), VIII(1)-IX(1) \\
\hline 193 & Lacanobia contigua (Denis \& Schiff., 1775) & $\mathrm{x}$ & $\mathrm{x}$ & $\mathrm{x}$ & $\mathrm{x}$ & $\mathrm{x}$ & VI(2),VII(1)-VIII(2) \\
\hline 194 & Lacanobia suasa (Denis \& Schiff., 1775) & $\mathrm{x}$ & $\mathrm{x}$ & $\mathrm{x}$ & $\mathrm{x}$ & $\mathrm{x}$ & VI(3)-VII(2), VIII(1)-IX(2) \\
\hline \multirow[t]{2}{*}{195} & Hada plebeja (Linnaeus, 1761) & & & & & & \\
\hline & = Hada nana (Hufnagel, 1766) & $\mathrm{x}$ & $\mathrm{x}$ & $\mathrm{x}$ & $\mathrm{x}$ & $\mathrm{x}$ & V(1)-VI(3), VII(2)-X(3) \\
\hline 196 & Hecatera bicolorata (Hufnagel, 1766) & & & & & $\mathrm{x}$ & $\mathrm{V}(1)$ \\
\hline 197 & Hadena compta (Denis \& Schiff., 1775) & & & & & $\mathrm{x}$ & VI(3) \\
\hline 198 & Hadena bicruris (Hufnagel, 1766) & $\mathrm{x}$ & $\mathrm{x}$ & $\mathrm{x}$ & $\mathrm{x}$ & $\mathrm{x}$ & VI(1-2), VII(1)-IX(1) \\
\hline 199 & Hadena perplexa (Denis \& Schiff., 1775) & & $\mathrm{x}$ & $\mathrm{x}$ & & & VI(1), VII(1)-VIII(2) \\
\hline 200 & Sideridis reticulata (Goeze, 1781) & & $\mathrm{x}$ & $\mathrm{x}$ & $\mathrm{x}$ & $\mathrm{x}$ & VI(1)-VII(1) \\
\hline 201 & Sideridis rivularis (Fabricius, 1775) & $\mathrm{x}$ & $\mathrm{x}$ & $\mathrm{x}$ & $\mathrm{x}$ & $\mathrm{x}$ & VI(1)-VII(3), X(3) \\
\hline \multirow[t]{2}{*}{202} & Sideridis turbida (Esper, 1790) & & & & & & \\
\hline & = Sideridis albicolon (Hübner, 1813) & $\mathrm{x}$ & $\mathrm{x}$ & $\mathrm{x}$ & $\mathrm{x}$ & $\mathrm{x}$ & $\mathrm{VI}(1-2)$ \\
\hline \multirow[t]{2}{*}{203} & Melanchra persicariae (Linnaeus, 1761) & $\mathrm{x}$ & $\mathrm{x}$ & $\mathrm{x}$ & $\mathrm{x}$ & $\mathrm{x}$ & VI(2)-VII(3) \\
\hline & Ceramica pisi (Linnaeus, 1758) & $\mathrm{x}$ & $\mathrm{x}$ & $\mathrm{x}$ & $\mathrm{x}$ & $\mathrm{x}$ & VI(1)-VII(3) \\
\hline 204 & Mamestra brassicae (Linnaeus, 1758) & $\mathrm{x}$ & & & $\mathrm{x}$ & $\mathrm{x}$ & VI(2), VIII(1)-X(3) \\
\hline 205 & Polia bombycina (Hufnagel, 1766) & & $\mathrm{x}$ & $\mathrm{x}$ & $\mathrm{x}$ & $\mathrm{x}$ & VII(1-2) \\
\hline 206 & Polia hepatica (Clerck, 1759) & & & & $\mathrm{x}$ & & VII(2) \\
\hline 207 & Polia nebulosa (Hufnagel, 1766) & $\mathrm{x}$ & $\mathrm{x}$ & $\mathrm{x}$ & $\mathrm{x}$ & $\mathrm{x}$ & VI(2)-VII(2) \\
\hline 208 & Mythimna turca (Linnaeus, 1758) & $\mathrm{x}$ & $\mathrm{x}$ & & $\mathrm{x}$ & $\mathrm{x}$ & VI(2)-VII(2) \\
\hline 209 & Mythimna conigera (Denis \& Schiff., 1775) & $\mathrm{x}$ & $\mathrm{x}$ & $\mathrm{x}$ & $\mathrm{x}$ & $\mathrm{x}$ & VI(3)-VII(3) \\
\hline 210 & Mythimna ferrago (Fabricius, 1787) & $\mathrm{x}$ & & & $\mathrm{x}$ & $\mathrm{x}$ & VI(3)-VIII(1) \\
\hline 211 & Mythimna albipuncta (Denis \& Schiff., 1775) & $\mathrm{x}$ & $\mathrm{x}$ & $\mathrm{x}$ & & $\mathrm{x}$ & VI(1-2), VIII(1)-X(3) \\
\hline 212 & Mythimna pudorina (Denis \& Schiff., 1775) & $\mathrm{x}$ & & $\mathrm{x}$ & $\mathrm{x}$ & $\mathrm{x}$ & VI(2)-VII(2) \\
\hline 213 & Mythimna straminea (Treitschke, 1825) & $\mathrm{x}$ & & & & $\mathrm{x}$ & VII(1)-VIII(2) \\
\hline 214 & Mythimna impura (Hübner, 1808) & $\mathrm{x}$ & $\mathrm{x}$ & & $\mathrm{x}$ & $\mathrm{x}$ & $\mathrm{VI}(1)-\mathrm{X}(3)$ \\
\hline 215 & Mythimna pallens (Linnaeus, 1758) & $\mathrm{x}$ & $\mathrm{x}$ & $\mathrm{x}$ & $\mathrm{x}$ & $\mathrm{x}$ & V(2)-VI(2), VII(2)-IX(2) \\
\hline 216 & Mythimna l-album (Linnaeus, 1767) & $\mathrm{x}$ & $\mathrm{x}$ & $\mathrm{x}$ & $\mathrm{x}$ & $\mathrm{x}$ & VI(2-3), VIII(2)-X(3) \\
\hline 217 & Leucania obsoleta (Hübner, 1803) & $\mathrm{x}$ & & $\mathrm{x}$ & & $\mathrm{x}$ & VI(1)-VII(3) \\
\hline 218 & Leucania comma (Linnaeus, 1761) & $\mathrm{x}$ & $\mathrm{x}$ & $\mathrm{x}$ & $\mathrm{x}$ & $\mathrm{x}$ & $\mathrm{VI}(1-3)$ \\
\hline 219 & Orthosia incerta (Hufnagel, 1766) & $\mathrm{x}$ & $\mathrm{x}$ & $\mathrm{x}$ & $\mathrm{x}$ & $\mathrm{x}$ & $\mathrm{IV}(2)-\mathrm{V}(1)$ \\
\hline 220 & Orthosia gothica (Linnaeus, 1758) & $\mathrm{x}$ & $\mathrm{x}$ & $\mathrm{x}$ & $\mathrm{x}$ & $\mathrm{x}$ & $\operatorname{IV}(2)-V(1)$ \\
\hline 221 & Orthosia cruda (Denis \& Schiff., 1775) & $\mathrm{x}$ & $\mathrm{x}$ & $\mathrm{x}$ & $\mathrm{x}$ & $\mathrm{x}$ & $\operatorname{IV}(2)-V(1)$ \\
\hline 222 & Orthosia miniosa (Denis \& Schiff., 1775) & $\mathrm{x}$ & & & & $\mathrm{x}$ & $\mathrm{V}(1)-\mathrm{V}(1)$ \\
\hline 223 & Orthosia opima (Hübner, 1809) & $\mathrm{x}$ & $\mathrm{x}$ & $\mathrm{x}$ & $\mathrm{x}$ & $\mathrm{x}$ & $\operatorname{IV}(2)$ \\
\hline 224 & Orthosia populeti (Fabricius, 1775) & $\mathrm{x}$ & $\mathrm{x}$ & $\mathrm{x}$ & $\mathrm{x}$ & $\mathrm{x}$ & $\operatorname{IV}(2)-V(1)$ \\
\hline 225 & Orthosia cerasi (Fabricius, 1775) & $\mathrm{x}$ & $\mathrm{x}$ & $\mathrm{x}$ & $\mathrm{x}$ & $\mathrm{x}$ & $\operatorname{IV}(2)$ \\
\hline
\end{tabular}




\begin{tabular}{|c|c|c|c|c|c|c|c|}
\hline \multirow[b]{2}{*}{ No. } & \multirow[b]{2}{*}{ Species } & \multicolumn{5}{|c|}{ Study site } & \multirow[b]{2}{*}{ Flight period* } \\
\hline & & 1 & 2 & 3 & 4 & 5 & \\
\hline 226 & Orthosia gracilis (Denis \& Schiff., 1775) & $\mathrm{x}$ & $\mathrm{x}$ & & & & $\mathrm{V}(1)$ \\
\hline 227 & Orthosia munda (Denis \& Schiff., 1775) & $\mathrm{x}$ & $\mathrm{x}$ & $\mathrm{x}$ & $\mathrm{x}$ & $\mathrm{x}$ & $\mathrm{IV}(2)-\mathrm{V}(1)$ \\
\hline 228 & Panolis flammea (Denis \& Schiff., 1775) & $\mathrm{x}$ & $\mathrm{x}$ & $\mathrm{x}$ & $\mathrm{x}$ & $\mathrm{x}$ & $\mathrm{IV}(2)-\mathrm{V}(1)$ \\
\hline 229 & Egira conspicillaris (Linnaeus, 1758) & $\mathrm{x}$ & $\mathrm{x}$ & & $\mathrm{x}$ & $\mathrm{x}$ & $\mathrm{V}(1-2)$ \\
\hline 230 & Cerapteryx graminis (Linnaeus, 1758) & $\mathrm{x}$ & $\mathrm{x}$ & $\mathrm{x}$ & $\mathrm{x}$ & $\mathrm{x}$ & VII(1)-VIII(2) \\
\hline 231 & Tholera cespitis (Denis \& Schiff., 1775) & $\mathrm{x}$ & $\mathrm{x}$ & $\mathrm{x}$ & $\mathrm{x}$ & $\mathrm{x}$ & VIII(2)-IX(2) \\
\hline 232 & Tholera decimalis (Poda, 1761) & $\mathrm{x}$ & $\mathrm{x}$ & & $\mathrm{x}$ & $\mathrm{x}$ & VIII(2)-IX(2) \\
\hline & Noctuinae & & & & & & \\
\hline 233 & Axylia putris (Linnaeus, 1761) & $\mathrm{x}$ & $\mathrm{x}$ & $\mathrm{x}$ & $\mathrm{x}$ & $\mathrm{x}$ & VI(1)-VII(3), X(3) \\
\hline 234 & Ochropleura plecta (Linnaeus, 1761) & $\mathrm{x}$ & $\mathrm{x}$ & $\mathrm{x}$ & $\mathrm{x}$ & $\mathrm{x}$ & VI(1-3), VII(2)-IX(2) \\
\hline 235 & Diarsia brunnea (Denis \& Schiff., 1775) & $\mathrm{x}$ & $\mathrm{x}$ & $\mathrm{x}$ & $\mathrm{x}$ & $\mathrm{x}$ & VI(2)-VII(3) \\
\hline 236 & Diarsia rubi (Vieweg, 1790) & $\mathrm{x}$ & $\mathrm{x}$ & & $\mathrm{x}$ & $\mathrm{x}$ & V(2)-VI(3), VIII(1-3) \\
\hline 237 & Diarsia florida (F. Schmidt, 1859) ** & & & & & $\mathrm{x}$ & $\mathrm{VI}(1-2)$ \\
\hline 238 & Noctua pronuba (Linnaeus, 1758) & $\mathrm{x}$ & $\mathrm{x}$ & $\mathrm{x}$ & $\mathrm{x}$ & $\mathrm{x}$ & $\mathrm{VI}(1)-\mathrm{X}(3)$ \\
\hline 239 & Noctua orbona (Hufnagel, 1766) & & $\mathrm{x}$ & & $\mathrm{x}$ & $\mathrm{x}$ & VI(2)-IX(1) \\
\hline 240 & Noctua interposita (Hübner, 1790) & & $\mathrm{x}$ & $\mathrm{x}$ & & $\mathrm{x}$ & $\mathrm{VI}(3)-\mathrm{IX}(1)$ \\
\hline 241 & Noctua comes Hübner, 1813 & & & & & $\mathrm{x}$ & $\operatorname{VII}(1)$ \\
\hline 242 & Noctua fimbriata (Schreber, 1759) & $\mathrm{x}$ & $\mathrm{x}$ & $\mathrm{x}$ & $\mathrm{x}$ & $\mathrm{x}$ & VI(3)-IX(2) \\
\hline 243 & Noctua janthina (Denis \& Schiff., 1775) & $\mathrm{x}$ & $\mathrm{x}$ & $\mathrm{x}$ & & $\mathrm{x}$ & VII(1)-VIII(1) \\
\hline 244 & Noctua janthe (Borkhausen, 1792) & $\mathrm{x}$ & $\mathrm{x}$ & & & $\mathrm{x}$ & VII(3)-VIII(1) \\
\hline 245 & Lycophotia molothina (Esper, 1789) ** & & & & & $\mathrm{x}$ & $\mathrm{V}(2)-\mathrm{VII}(2)$ \\
\hline 246 & Lycophotia porphyrea (Denis \& Schiff., 1775) & $\mathrm{x}$ & $\mathrm{x}$ & $\mathrm{x}$ & & $\mathrm{x}$ & VII(1)-IX(1) \\
\hline 247 & Rhyacia simulans (Hufnagel, 1766) & & $\mathrm{x}$ & & & & VII(2-3) \\
\hline 248 & Paradiarsia punicea (Hübner, 1803) ** & & $\mathrm{x}$ & & & & $\mathrm{VI}(3)$ \\
\hline 249 & Eurois occulta (Linnaeus, 1758) & $\mathrm{x}$ & $\mathrm{x}$ & & $\mathrm{x}$ & $\mathrm{x}$ & VI(2)-VIII(1) \\
\hline 250 & Graphiphora augur (Fabricius, 1775) & & & $\mathrm{x}$ & & & VII $(1-2)$ \\
\hline 251 & Protolampra sobrina (Duponchel, 1843) & $\mathrm{x}$ & $\mathrm{x}$ & & $\mathrm{x}$ & $\mathrm{x}$ & VII(2)-VIII(3) \\
\hline 252 & Xestia c-nigrum (Linnaeus, 1758) & $\mathrm{x}$ & $\mathrm{x}$ & $\mathrm{x}$ & $\mathrm{x}$ & $\mathrm{x}$ & VI(1)-VII(2), VIII(2)-X(3) \\
\hline 253 & Xestia ditrapezium (Denis \& Schiff., 1775) & $\mathrm{x}$ & $\mathrm{x}$ & $\mathrm{x}$ & $\mathrm{x}$ & & VI(2)-VII(3) \\
\hline 254 & Xestia triangulum (Hufnagel, 1766) & $\mathrm{x}$ & $\mathrm{x}$ & & $\mathrm{x}$ & $\mathrm{x}$ & VI(1)-VII(2) \\
\hline 255 & Xestia baja (Denis \& Schiff., 1775) & $\mathrm{x}$ & $\mathrm{x}$ & $\mathrm{X}$ & $\mathrm{x}$ & $\mathrm{x}$ & VII(1)-IX(2) \\
\hline 256 & Xestia rhomboidea (Esper, 1790) & & & & $\mathrm{x}$ & & VII(2-3) \\
\hline 257 & Xestia castanea (Esper, 1798) ** & & & & & $\mathrm{x}$ & 30 VII 2013 \\
\hline 258 & Xestia sexstrigata (Haworth, 1809) & $\mathrm{x}$ & $\mathrm{x}$ & & $\mathrm{x}$ & $\mathrm{x}$ & VIII $(1-3)$ \\
\hline 259 & Xestia xanthographa (Denis \& Schiff., 1775) & $\mathrm{x}$ & $\mathrm{x}$ & & $\mathrm{x}$ & $\mathrm{x}$ & VIII(2)-IX(2) \\
\hline 260 & Eugraphe sigma (Denis \& Schiff., 1775) & & $\mathrm{x}$ & & $\mathrm{x}$ & $\mathrm{x}$ & VI(2)-VII(3) \\
\hline 261 & Coenophila subrosea (Stephens, 1829) ** & & & & & $\mathrm{x}$ & VIII(1)-IX(2) \\
\hline 262 & Cerastis rubricosa (Denis \& Schiff., 1775) & $\mathrm{x}$ & $\mathrm{x}$ & $\mathrm{x}$ & $\mathrm{x}$ & $\mathrm{x}$ & $\operatorname{IV}(2)-V(1)$ \\
\hline 263 & Naenia typica (Linnaeus, 1758) & $\mathrm{x}$ & & & & & $\operatorname{VII}(2)$ \\
\hline 264 & Anaplectoides prasina (Denis \& Schiff., 1775) & $\mathrm{x}$ & $\mathrm{x}$ & $\mathrm{x}$ & $\mathrm{x}$ & $\mathrm{x}$ & VI(1)-VII(1) \\
\hline 265 & Actebia praecox (Linnaeus, 1758) & & & & $\mathrm{x}$ & & VII(2-3) \\
\hline 266 & Euxoa nigricans (Linnaeus, 1761) & $\mathrm{x}$ & & $\mathrm{x}$ & & $\mathrm{x}$ & VIII(1)-IX(1) \\
\hline 267 & Euxoa tritici (Linnaeus, 1761) & $\mathrm{x}$ & $\mathrm{x}$ & $\mathrm{x}$ & $\mathrm{x}$ & $\mathrm{x}$ & VII(3)-IX(1) \\
\hline 268 & Euxoa vitta (Esper, 1789) ** & & & & & $\mathrm{x}$ & 8 VIII 2013 \\
\hline 269 & Agrotis ipsilon (Hufnagel, 1766) & $\mathrm{x}$ & $\mathrm{x}$ & $\mathrm{x}$ & & $\mathrm{x}$ & $\mathrm{VI}(2)-\mathrm{X}(3)$ \\
\hline 270 & Agrotis exclamationis (Linnaeus, 1758) & $\mathrm{x}$ & $\mathrm{x}$ & $\mathrm{x}$ & $\mathrm{x}$ & $\mathrm{x}$ & VI(1)-VII(3) \\
\hline 271 & Agrotis segetum (Denis \& Schiff., 1775) & $\mathrm{x}$ & $\mathrm{x}$ & $\mathrm{x}$ & & $\mathrm{x}$ & VI(2),VIII(1)-IX(2) \\
\hline 272 & Agrotis vestigialis (Hufnagel, 1766) & $\mathrm{x}$ & $\mathrm{x}$ & $\mathrm{x}$ & $\mathrm{x}$ & $\mathrm{x}$ & VII(1)-IX(2) \\
\hline 273 & Agrotis cinerea (Denis \& Schiff., 1775) & & & & & $\mathrm{x}$ & $\mathrm{V}(1)$ \\
\hline
\end{tabular}




\section{Idia calvaria (Denis \& Schiff., 1775)}

An Asian-Mediterranean species, it occurs more numerously in southern Europe. Records from Poland to date are from single sites, mainly in the southern and eastern part of the country (Buszko \& Nowacki 2000). In the second half of the 20th century it was confirmed only from the Przemyśl Foothills (Bielewicz 1973), the Kraków area (Razowski, Palik 1969) and the Białowieża Primeval Forest (Buszko et al. 1996).

During this research one specimen was caught in a light trap at the Buda Stalowska site on 9 June 2013.

\section{Meganola togatulalis (Hübner, 1796)}

This is a species with a European distribution, reported in small numbers from all over central Europe. In Poland it is very rare, but it has been recorded at single sites all over the country (Buszko \& Nowacki 2000).

During this study it was reported only from the beginning to the end of July 2013 in Nowa Dęba on a dune heath with single oak trees, birches and barberry bushes. In this ecosystem it was very numerous, often with several dozen specimens in a single catch.

\section{Diachrysia zosimi (Hübner, 1822)}

A very rare Euro-Siberian species, occurring only locally in its distribution range in Europe. It has been reported only from single, extremely isolated sites, often several hectares in area. It occurs only in fens and transitional bogs. The number of its known sites throughout its European range has fallen dramatically (Nowacki 1998). A strongly stenotopic species, it occurs in Poland at a few sites, mainly in the north and east of the country: the Biebrza Marshes (Frąckiel \& Nowacki 2010), the Augustów Primeval Forest (Nowacki 1989), the Białowieża Primeval Forest (Buszko et al. 1996), the Chełm area (Buszko et al. 1996a), and the Polesie (Nowacki \& Hołowiński 1999) and Roztocze regions (Nowacki \& Pałka 2013).

During this study, just one single specimen was caught in a light trap in wet meadows near the forester's lodge on 18 June 2013 at Buda Stalowska.

\section{Syngrapha microgamma (Hübner, 1823)}

This Boreal species with a Holarctic range is extremely rare, occurring only in raised bogs and marshy coniferous forests. Its range in Europe has contracted greatly and is now found only at very isolated sites. In Poland it has become extinct in the western part of the country. At present it occurs at scattered sites in eastern Poland and in the Podhale region of southern Poland (Buszko \& Nowacki 2000).

During the present study, one specimen was caught in a light trap at Krawce in mid-June.

\section{Abrostola asclepiadis (Denis \& Schiff., 1775)}

This is a southern European species whose range extends into central Europe. It is characteristic of the dry, warm and open ecosystems of xerothermic or psammophilous grasslands. It occurs locally at single sites in southern and eastern Poland (Buszko \& Nowacki 2000).

During this study it occurred only in early June 2013 at the Nowa Dęba site: single specimens were caught in light traps deployed in psammophilous grasslands and dune heaths. 


\section{Acronicta menyanthidis (Esper, 1789)}

This is a Euro-Siberian, stenotopic species that occurs at a few scattered sites, mainly in northern and eastern Poland. As a consequence of the degradation of peat bog ecosystems in the second half of the $20^{\text {th }}$ century, its range of occurrence has contracted significantly. In recent years the species has been confined mainly to eastern Poland: the Biebrza Marshes (Frąckiel \& Nowacki 2010), the Sobibór Forests (Nowacki \& Hołowiński 1999), the Polesie National Park (Nowacki \& Pałka 2013), the Augustów Primeval Forest (Nowacki \& Rudny 1992), the Białowieża Primeval Forest (Buszko et al. 1996), the Romincka Primeval Forest (Nowacki 1992a) and the Roztocze region (Nowacki 1992).

During this study single specimens were caught in light traps deployed in peat bogs but only early and mid July at the Bojanów and Nowa Dęba sites.

\section{Heliothis ononis (Denis \& Schiff., 1775)}

This is a Holarctic species of the steppe zone, whose European range extends westwards as far as Austria, Switzerland and France. Very rare in Poland, it has been reported from a handful of sites, mainly in the southern part of the country. In recent years it has been reported only from the Lublin area (Nowacki \& Hołowiński 1999).

During this study single specimens were caught during the first 20 days of June 2013 at the Huta Komorowska site.

\section{Hoplodrina respersa (Denis \& Schiff., 1775)}

A Holo-Mediterranean species, widespread throughout Europe. In Poland, however, it occurs only locally at a few, often isolated sites, particularly in the south (Buszko \& Nowacki 2000). It is a stenotopic species that inhabits xerothermic grasslands, including psammophilous swards.

In this study one specimen was caught on 3 August 2013 at Nowa Dęba in the psammophilous sward and dune heath habitat.

\section{Lithophane lamda (Fabricius, 1787)}

A very rare Boreal species with a Holarctic distribution, it occurs only in peat bogs and marshy coniferous forests. Its central European range has shrunk considerably. It no longer occurs in western and central Poland: there are historical records from Pomerania, Silesia, Warsaw and Sandomierz (Buszko \& Nowacki 2000). Currently it occurs in Poland in single scattered localities on the Baltic coast (Nowacki 1994), in the Augustów Primeval Forest (Nowacki \& Rudny 1992), the Białowieża Primeval Forest (Buszko et al. 1996), the Janów Forests, the Polesie (Nowacki \& Hołowiński 1999) and Roztocze regions (Nowacki 2006), and the Solska Primeval Forest (Nowacki \& Pałka 2013)

During the study single specimens were caught in light traps deployed in peat bogs and marshy coniferous forests at Krawce and Nowa Dęba from mid to late April 2013.

\section{Xylomoia graminea (Graeser, 1889)}

A Euro-Siberian species first reported from Europe in 1988 - in fact, from the Roztocze region of south-eastern Poland (Nowacki 1989). Currently it occurs in central Europe only in disjunctive localities as far as the line of the Vistula. In Poland it has been so far reported from the Biebrza Marshes (Frąckiel \& Nowacki 2010), Podlasie (Nowacki \& Wasiluk 2004), Polesie (Nowacki \& Hołowiński 1999), the Augustów Primeval Forest (Kokot 1995), the Białowieża Primeval Forest (Wąsala 2001), the Roztocze region (Nowacki 1992), the Lublin Upland, Bąkowiec and Janowice in the Vistula valley, the Janów Forests and Nowy Lubliniec and 
Starzawa in the Sandomierz Basin (Nowacki \& Pałka 2013), and the Świętokrzyskie Mts. (Nowacki \& Nowacka 2012). Its populations in Poland live at the westernmost extremity of its distribution range and are among the most abundant in Europe.

During the study it was light-trapped at the Nowa Dęba site in early June 2013, and at Nowy Lubliniec and Starzawa in earlier years.

\section{Hydraecia ultima (Holst, 1965)}

A Euro-Siberian species with central Europe as the western boundary of its distribution range. In Poland it has been reported from just a few localities mainly in the south and east of the country, although it was also found in the Żary area (south-western Poland) (Nowacki et al. 1997).

During the study it was found from mid-July to the first days of August 2013 at the sites in Bojanowo, Buda Stalowska and Krawce, where single specimens were caught in wetland habitats.

\section{Diarsia florida (F. Schmidt, 1859)}

A Euro-Siberian species characteristic of marshes, peat bogs and wet meadows. For a long time it was not distinguished from $D$. rubi, so the data on its occurrence in Poland are not exact. It occurs all over the country, but only in isolated localities of a suitable ecological type (Buszko \& Nowacki 2000).

During the study one specimen was caught in wet meadows at Nowa Dęba in the first half of June 2013.

\section{Lycophotia molothina (Esper, 1789)}

An Atlantic species with Poland at the easternmost end of its range. So far it has been reported from single sites in western and southern Poland, and as far east as far as the Lublin province. There are recent records from Lower Silesia and Lubuskie province, and also single specimens from Nowa Dęba (Mazurkiewicz \& Pałka 2003) and the Janów Forests (Nowacki \& Pałka 2013).

During the study it was found from mid-May to 20 July only at the Nowa Dęba site, at many localities on dunes, in open heathland and dry coniferous forests. In these ecosystems it was very numerous, with dozens of specimens frequently being caught during a single trapping session.

\section{Paradiarsia punicea (Hübner, 1803)}

A Euro-Siberian species with the boundary of its contiguous range in north-eastern Poland. Elsewhere in central Europe it occurs only in scattered and disjunctive localities. Being a stenotopic species, it occurs only in peat bogs and marshes and is always very rare. In Poland it occurs very locally. The sites confirmed to date include the Biebrza Marshes (Frąckiel \& Nowacki 2010), the Augustów Primeval Forest (Nowacki \& Rudny 1992), the Białowieża Primeval Forest (Buszko et al. 1996), the Sobibór Forests (Nowacki \& Hołowiński 1999), the Chełm area (Buszko et al. 1996a) and the Polesie National Park, the middle Vistula Valley around Bąkowiec, and the Zamość area (Nowacki \& Pałka 2013).

During the study it was found only in late June 2013 at Krawce, where one specimen was caught in a light trap.

\section{Xestia castanea (Esper, 1798)}

An Atlantic-Mediterranean species found in a few localities in Poland, mainly in the west of the country. Historically it was reported from Western Pomerania (Urbahn 1939), Lower Silesia (Wolf 1935), the Zawiercie area (Masłowski 1936) and Gocławek (Patryn 1938). More 
recently, it has been confirmed from single localities on the Baltic coast as far as Hel (Nowacki 1994) and in western Poland (Buszko \& Nowacki 2000), and also in the Janów Forests in south-eastern Poland (Nowacki \& Pałka 2013).

During the study one specimen was caught in a light trap in open heathland at Nowa Dęba on 30 August 2013.

\section{Coenophila subrosea (Stephens, 1829)}

A Euro-Siberian species, characteristic of peat bogs, marshes and wet meadows. In western and central Europe it occurs very locally at a few sites, the numbers of which are decreasing. A stenotopic species, it is very local in Poland. Historically it was reported from Western Pomerania (Urbahn 1939). It is currently found in the Biebrza Marshes (Frackiel \& Nowacki 2010), the Augustów Primeval Forest (Nowacki \& Rudny 1992), the Białowieża Primeval Forest (Buszko et al. 1996), the Sobibór Forests (Nowacki \& Hołowiński 1999), the Polesie National Park, the Chełm area (Nowacki \& Pałka 2013) and the Roztocze region (Nowacki 1992). The Polish populations, particularly those of the Biebrza Marshes, are among the most numerous in Europe.

During the study, it was found from the beginning of August to mid-September 2013 at the "Cietrzewiec" site in Nowa Dęba and the surrounding wet meadows, where several dozen specimens were caught in light traps.

\section{Euxoa vitta (Esper, 1789)}

An Asian-Mediterranean species, with a contiguous range in southern Europe; in central Europe it now occurs only at isolated sites in xerothermic grasslands. In Poland it has been reported only from the Ponidzie and Wielkopolska regions (Nowacki \& Śliwa 1996).

During the study one specimen was caught on 8 August 2013 at the Nowa Dęba locality, in a light trap deployed in the psammophilous grasslands and dune heaths.

\section{SUMMARY OF RESULTS}

Extensive, natural marshy river valleys are unique in western Europe and are disappearing in east-central Europe. The field study of 1999-2013, covering various ecosystems in the Sandomierz Primeval Forest, has enabled us to update our knowledge of the Noctuoidea inhabiting this area.

273 species from this superfamily were found in the Nowa Dęba Military Training Area, i.e. about 55\% of all the Noctuoidea so far reported from Poland. The number of these moths in the Sandomierz Primeval Forest confirmed in the current study cannot be treated as definitive, however, as indicated by the species richness of this group in other natural and geographical regions of eastern Poland, such as the Biebrza Marshes - 299 species (Frąckiel \& Nowacki 2010), the Augustów Primeval Forest - 323 species (Nowacki \& Rudny 1992, Nowacki 2006, Kokot 1995), the Sobibór Forests in Polish Polesie - 337 species (Nowacki \& Hołowiński 1999, 2014), central Podlasie - 319 species (Nowacki \& Wasiluk 2004) and the Białowieża Primeval Forest - 352 species (Buszko et al. 1996, Wąsala 2001).

The Noctuidae reported from the Sandomierz Primeval Forest include species of faunistic importance for both Poland and Europe. They occur rarely or very locally in Poland and are most often stenotopic. A total of 18 noctuid species found in the Sandomierz Primeval Forest are considered faunistically valuable in Poland. Making up 6.3\% of the noctuid species reported from the study area, they include: I. calvaria, M. togatulalis, D. zosimi, S. microgamma, A. asclepiadis, A. menyanthidis, $H$. ononis, $H$. respersa, L. lamda, A. caecimacula, X. graminea, $H$. ultima, $D$. florida, L. molothina, P. punicea, X. castanea, C. subrosea and E. vitta. 
Analysis of the material collected during the study enabled these noctuids to be characterised on the basis of their assemblages in the particular plant communities of the Nowa Dęba Military Training Area. The species composition of Noctuidae in wetland habitats is of particular interest. Such habitats consist mainly of fens and transitional peat bogs, swathes of bulrushes around fishponds and the numerous drainage ditches, and marshy coniferous forests and alder swamps. These habitats support the largest number of stenotopic species. Among all the Noctuidae species considered rare, there are 19 hygrophilous ones: A. menyanthidis, $A$. cinerea, $H$. humidalis, $D$. zosimi, S. microgamma, X. graminea, L. lamda, C. maritima, A. unanimis, A. lucens, H. ultima, $P$. nexa, A. dissoluta, S. buettneri, M. straminea, O. opima, D. florida, P. punicea and C. subrosea.

By comparison, the numbers of hygrophilous noctuid species reported from other natural and geographical areas of eastern Poland are as follows: the Biebrza Marshes (21 species), the Białowieża Primeval Forest (19), the Augustów Primeval Forest (14), the Sobibór Forests in Polish Polesie (13) and central Podlasie (9). These species are mainly stenotopic ones that inhabit strictly defined ecosystems, mainly of a natural character. The data presented in this paper relating to hygrophilous species emphasise the importance of the Sandomierz Primeval Forest marshes for preserving the biodiversity of this Noctuidae group in Poland.

The psammophilous and xerothermic noctuid species of the numerous sand dune ecosystems in the Sandomierz Primeval Forest, particularly the Nowa Dęba Military Training Area, are also worth noting. They include $M$. togatulalis, A. asclepiadis, S. nervosa, C. artemisiae, Sh. verbasci, C. lunula, P. scutosa, $H$. ononis, C. fraudatricula, $H$. respersa, A. caecimacula, $C$. tridens, A. myrtilli, $H$. compta, $H$. perplexa, L. molothina, L. porphyrea, $X$. castanea, A. praecox, $E$. vitta and $A$. cinerea. They make up a large proportion (7.7\%) of the noctuid assemblage of the Sandomierz Primeval Forest. At the same time, they are a valuable faunistic element, adding to the region's and the country's biodiversity.

The conclusion to be drawn from the results of this study are that the Nowa Dęba Military Training Area in the Sandomierz Primeval Forest constitutes an important refugium for both psammophilous/xerothermic and wetland noctuids and is of crucial significance for biodiversity conservation in both Poland and Europe.

\section{REFERENCES}

BIELEwiCz M., 1973. Motyle Bieszczadów Zachodnich i Pogórza Przemyskiego, Cz. I tzw. Macrolepidoptera. Roczniki Muzeum Górnośląskiego w Bytomiu., Przyroda, 7: 1-170.

BIELEWICZ M., 1984. Nowe gatunki motyli większych (Macrolepidoptera) dla fauny Bieszczadów Zachodnich i Pogórza Przemyskiego. Polskie Pismo Entomologiczne 54: 407-409.

Buszko J., KoкOT A., PALIK E. \& ŚliwiŃski Z.,1996. Motyle większe (Macrolepidoptera) Puszczy Białowieskiej. Parki Narodowe i Rezerwaty Przyrody, Białowieża, 5 (4): 3-46.

Buszko J., Kaitila J. P., Junnilainen J., NowACKi J., NupPOnen K. \& PAŁKa K. 1996a. Nowe i rzadko spotykane gatunki motyli w faunie Polski. Wiadomości Entomologiczne 15: 105-115.

BuszKo J. \& NowACKI J., 2000. The Lepidoptera of Poland. A Distributional Checklist. Polish Entomological Monographs, 1: $178 \mathrm{pp}$.

FrĄCKIEL K. \& NOWACKI J. 2010. The Noctuids (Lepidoptera, Noctuidae) of marsh ecosystems in the Biebrza National Park. Polish Entomological Monographs 7: 67 pp.

HoŁowIŃSKI M. \& NowACKI J. 2014. Sówkowate (Lepidoptera: Noctuidae) nowe dla Lasów Sobiborskich na obszarze Polskiego Polesia. Wiadomości Entomologiczne 33: 75-76.

KARPOWICZ S. 1928. Spis łuskoskrzydłych Ziemi Sandomierskiej II. Polskie Pismo Entomologiczne 7: 93-122.

Kокот A. 1995: Nowe dla Puszczy Augustowskiej gatunki sówkowatych (Lepidoptera, Noctuidae). Wiadomosci Entomologiczne 14: 62.

KONDRACKI J. 1998. Geografia regionalna Polski. PWN, Warszawa, 441 pp.

Kulesza W. 1936. Przyczynek do znajomości fauny motyli niektórych okolic Polski. Sprawozdania Komsji Fizjograficznej, PAU 70: 139-148.

MASŁOWSKI L. \& MASŁOWSKI M. 1936: Motyle okolic Zawiercia, III. Uzupełnienia i sprostowania. Fragmenta Faunistica Musei Zoologici Polonici 2: 403-450. 
MAZURKIEWICZ A. \& PAŁKA K. 2003: Nowe stanowiska rzadkich gatunków sówkowatych (Lepidoptera, Noctuidae) w Polsce. Wiadomosci Entomologiczne 22: 251-252.

NOWACKI J. 1992. Sówkowate (Lepidoptera, Noctuidae) Roztocza. Fragmenta Faunistica 35, 24: $397-414$.

NOWACKI J. 1992a: Materiały do poznania sówkowatych (Lepidoptera, Noctuidae) Puszczy Rominckiej. Wiadomości entomologiczne 11: 113-119.

NowACKI J. \& RuDNy J., 1992. Sówkowate (Lepidoptera, Noctuidae) Puszczy Augustowskiej. Wiadomości Entomologiczne 11 (1): $37-57$.

NOWACKI J., 1994. Struktura zasięgów sówkowatych (Lepidoptera, Noctuidae) w pasie wydm nadmorskich polskiego wybrzeża Bałtyku. Wiadomości Entomologiczne 12, Suppl.: 1-127.

NowACKI J. \& ŚlLIWA W., 1996. Nowe stanowisko Euxoa vitta (Esp.) (Lepidoptera, Noctuidae) w Polsce. Wiadomości Entomologiczne 15: 124.

NOWACKI J., FuglewiCZ E. \& FuglewiCZ S. 1997: Nowe stanowiska interesujących gatunków sówkowatych (Lepidoptera: Noctuidae) w zachodniej Polsce. Wiadomości Entomologiczne 16: 57.

NowACKI J., 1998. The Noctuids (Lepidoptera, Noctuidae) of central Europe. František Slamka, Bratislava, 51 pp.

NowACKI J. \& HoŁowiŃski M. 1999. Sówkowate (Lepidoptera: Noctuidae) Lasów Sobiborskich na obszarze Polskiego Polesia. Wiadomości Entomologiczne18, Supl.: 3-60.

NOWACKI J. \& WASILUK D. 2004: Sówkowate (Lepidoptera, Noctuidae) środkowego Podlasia. Wiadomości Entomologiczne 23 Suplement 1: 1-54.

NOWACKI J., 2006. Materiały do poznania sówek (Lepidoptera: Noctuoidea: Nolidae, Erebidae, Noctuidae) wschodniej Polski. Wiadomości Entomologiczne 25: 175-178.

NOWACKI J. \& NOWACKA J. 2012: Materiały do poznania sówkowatych (Lepidoptera: Noctuidae) Gór Świętokrzyskich. Wiadomości Entomologiczne 31: 35-40.

NOWACKI J. \& PAŁKA K. 2013. Materiały do poznania rozsiedlenia sówkowatych (Lepidoptera, Noctuidae) wschodniej Polski. Wiadomości Entomologiczne 32: 139-146.

PATRYN W. 1937-1938: Wzmianki motylnicze, II. Polksie Pismo Enomologiczne 16-17: 294-295.

RAZOWSKI J. \& PALIK E., 1969: Fauna motyli okolic Krakowa. Acta Zoologica Cracoviensis 14 (11): $217-310$.

URBAHN E ET H. 1939: Die Schmetterlinge Pommerns mit einem vergleichenden Überblick über den Ostseeraum. Entomologische Zeitung Stettin 100: 181-826.

WĄSALA R. 2001: Xylomoia graminea (Graeser) (Lepidoptera, Noctuidae) gatunek nowy dla Puszczy Białowieskiej. Wiadomości Entomologiczne 20: 95.

WoLF P. 1935: Die Grosschmetterlinge Schlesiens, II. Breslau. 161 - 341.

Woś A. 1999: Klimat Polski. PWN. Warszawa, 302 pp.

\section{STRESZCZENIE}

\section{[Sówkowate (Lepidoptera: Noctuoidea: Nolidae, Erebidae, Noctuidae) poligonu wojskowego “Nowa Deba” w Puszczy Sandomierskiej (południowo-wschodnie Polska)]}

W pracy zaprezentowano wyniki badań nad fauną sówek (Lepidoptera, Noctuoidea: Nolidae, Erebidae, Noctuidae) ekosystemów występujących na obszarze poligonu wojskowego „Nowa Dęba" na terenie Puszczy Sandomierskiej, przeprowadzonych w latach 1999-2013. Na badanym terenie stwierdzono występowanie 273 gatunków sówek. Stanowi to około 55\% wszystkich dotychczas wykazanych z Polski gatunków z badanych rodzin motyli. Uzyskane wyniki badań pozwalają na stwierdzenie, że poligon wojskowy „Nowa Dęba” na obszarze Puszczy Sandomierskiej stanowi ważne refugium dla psammofilnej i kserotermofilnej oraz higrofilnej fauny sówek i ma pierwszorzędne znaczenie dla ochrony różnorodności biologicznej Polski i Europy. Wśród Noctuidae wykazanych z Puszczy Sandomierskiej znajdują się gatunki rzadko spotykane lub występujące w kraju bardzo lokalnie. Łącznie z obszaru Puszczy Sandomierskiej wykazano 18 takich gatunków. Stanowią one 6,3\% liczby gatunków sówek wykazanych z obszaru badań. Do gatunków takich należą: I. calvaria, M. togatulalis, D. zosimi, S. microgamma, A. asclepiadis, A. menyanthidis, $H$. ononis, $H$. respersa, $L$. lamda, A. caecimacula, $X$. graminea, $H$. ultima, $D$. florida, $L$. molothina, $P$. punicea, $X$. castanea, $C$. subrosea oraz $E$. vitta. Gatunki te są w większości stenotopami występującymi w ściśle określonych ekosystemach, najczęściej o charakterze naturalnym. Stanowią one cenny element faunistyczny wzbogacający różnorodność biologiczną regionu i Polski. 